Bull. Korean Math. Soc. 52 (2015), No. 2, pp. 685-697

http://dx.doi.org/10.4134/BKMS.2015.52.2.685

\title{
ON HYERS-ULAM STABILITY OF NONLINEAR DIFFERENTIAL EQUATIONS
}

\author{
Jinghao Huang, Soon-Mo Jung, And YongJin Li
}

\begin{abstract}
We investigate the stability of nonlinear differential equations of the form $y^{(n)}(x)=F\left(x, y(x), y^{\prime}(x), \ldots, y^{(n-1)}(x)\right)$ with a Lipschitz condition by using a fixed point method. Moreover, a Hyers-Ulam constant of this differential equation is obtained.
\end{abstract}

\section{Introduction and preliminaries}

In 1940, S. M. Ulam [49] posed the following question concerning the stability of group homomorphisms before a Mathematical Colloquium: When can we assert that the solutions of an inequality are close to one of the exact solutions of the corresponding equation?

A year later, D. H. Hyers [15] dealt with $\varepsilon$-additive mapping by direct method, which gave a partial solution to the above question. The result was extended by T. Aoki [2], D. G. Bourgin [4] and Th. M. Rassias [40]. We mention here that the interest of this topic has been increasing since it came into being, some other results concerning functional equations one can find, e.g., in $[9,10,11,19,37,42,43]$ and some related information (e.g., $\varepsilon$-isometries, superstability of functional equations and the stability of differential expressions) we refer to $[3,5,6,8,14,16,17,18,36,47]$.

To the best of our knowledge, the first one who pay attention to the stability of differential equations is M. Obłoza [34, 35]. Thereafter, C. Alsina and R. Ger [1] proved that the stability holds true for differential equation $y^{\prime}(x)=y(x)$. Then, a generalized result was given by S.-E. Takahasi, T. Miura and S. Miyajima [48], in which they investigated the stability of the Banach space valued linear differential equation of first order (see also [31, 33]). A more general result on the linear differential equations of first order of the form $y^{\prime}(t)+\alpha(t) y(t)+\beta(t)=0$ was given by S.-M. Jung [22] and the stability of linear differential equations of second order was established by Y. Li et al. (see $[12,27,28,29])$. There are a number of results concerning the stability of the

Received May 27, 2014

2010 Mathematics Subject Classification. 34D20, $26 \mathrm{D} 10$.

Key words and phrases. Hyers-Ulam stability, generalized Hyers-Ulam stability, nonlinear differential equations, fixed point theorem. 
linear ordinary differential equations, which prompts the question: Can we assert that all of the linear ordinary differential equations have the Hyers-Ulam stability?

Indeed, P. Găvruţă, S.-M. Jung and Y. Li [12] proved that the differential equation $y^{\prime \prime}=0$ does not have the Hyers-Ulam stability on the whole domain. For some examples of differential equations which have the Hyers-Ulam stability on unbounded interval we refer the reader to $[1,20,32,38]$, which show that it is a very special case that the Hyers-Ulam stability holds true for general differential equations on the whole domain.

Recently, Jung [44] proved that the generalized Hyers-Ulam stability holds for the case of general linear differential equations, and the stability of nonlinear differential equations

$$
y^{\prime}(x)=F(x, y(x))
$$

with a Lipschitz condition on a local interval was investigated by a fixed point method:

Theorem $1.1([23])$. Given $c \in \mathbb{R}$ and $r>0$, let $I=[c-r, c+r]$ and let $F: I \times \mathbb{R} \rightarrow \mathbb{R}$ be a continuous function that satisfies a Lipschitz condition

$$
|F(x, y)-F(x, z)| \leq L|y-z|
$$

for all $x \in I$ and $y, z \in \mathbb{R}$, where $L$ is a constant with $0<L r<1$. Then the differential equation $y^{\prime}(x)=F(x, y(x))$ has the Hyers-Ulam stability with a Hyers-Ulam stability constant $\frac{r}{1-L r}$.

And then he raised an open question whether the differential equation $y^{\prime}(x)$ $=F(x, y(x))$ has the Hyers-Ulam stability when the relevant domain is an infinite interval.

In this paper, we adopt the ideas of V. Radu [39], S.-M. Jung [23] and Y. Li $[27,29]$ to investigate the generalized Hyers-Ulam stability as well as the Hyers-Ulam stability of the nonlinear differential equations of the form

$$
y^{(n)}(x)=F\left(x, y(x), y^{\prime}(x), \ldots, y^{(n-1)}(x)\right)
$$

on an interval $[a, b]$, where $n \in \mathbb{N}^{+}$, and the question raised by Jung [23] will be answered incidentally.

We now introduce the definition of the generalized metric on a nonempty set $\mathcal{X}$.

Definition 1.2. A function $d: \mathcal{X} \times \mathcal{X} \rightarrow[0, \infty]$ is called a generalized metric on $\mathcal{X}$ if

(i) $d(x, y)=0$ if and only if $x=y$;

(ii) $d(x, y)=d(y, x)$ for all $x, y \in \mathcal{X}$;

(iii) $d(x, z) \leq d(x, y)+d(y, z)$ for all $x, y, z \in \mathcal{X}$.

The following theorem is a very useful tool for proving our main theorems. 
Theorem $1.3([7])$. Let $(\mathcal{X}, d)$ be a generalized complete metric space and let $T: \mathcal{X} \rightarrow \mathcal{X}$ be a strictly contractive operator with the Lipschitz constant $L<1$. If there is a nonnegative integer $k$ such that $d\left(T^{k+1} x, T^{k} x\right)<\infty$ for a given $x \in \mathcal{X}$, then

(i) The sequence $\left\{T^{n} x\right\}_{n}$ converges to a fixed point $x^{*}$ of $T$;

(ii) $x^{*}$ is the unique fixed point of $T$ in

$$
\mathcal{X}^{*}=\left\{y \in \mathcal{X} \mid d\left(T^{k} x, y\right)<\infty\right\}
$$

(iii) For every $y \in \mathcal{X}^{*}$, it holds that

$$
d\left(y, x^{*}\right) \leq \frac{1}{1-L} d(T y, y)
$$

\section{Main results}

In this section, we investigate the generalized Hyers-Ulam stability and the Hyers-Ulam stability of differential equation (1.1).

For a given closed interval $I:=[a, b]$ and $\varphi(x) \in C\left(I, \mathbb{R}^{+}\right)$, we define a set

$$
\mathcal{X}:=\{f: I \rightarrow \mathbb{R} \mid f \text { is }(n-1) \text { times continuously differentiable }\}
$$

equipped with the metric

$d\left(y_{1}, y_{2}\right):=\inf \left\{C \in[0, \infty]\left|\max _{0 \leq i \leq n-1}\right| y_{1}^{(i)}(x)-y_{2}^{(i)}(x) \mid \leq C \varphi(x)\right.$ for every $\left.x \in I\right\}$.

Proposition 2.1. $\mathcal{X}$ is a complete generalized metric space.

Proof. We prove that $(\mathcal{X}, d)$ is a generalized metric space. For all $f, g, h \in \mathcal{X}$, we have

(i) $d(f, g)=0$ if and only if $|f(x)-g(x)|=\left|f^{\prime}(x)-g^{\prime}(x)\right|=\cdots=\mid f^{(n-1)}(x)-$ $g^{(n-1)}(x) \mid=0$ for every $x \in I$, which is equivalent to $f(x)=g(x)$ for each $x \in I$;

(ii) Since $\max _{0 \leq i \leq n-1}\left|f^{(i)}(x)-g^{(i)}(x)\right|=\max _{0 \leq i \leq n-1}\left|g^{(i)}(x)-f^{(i)}(x)\right|$, it holds that $d(f, g)=d(g, f)$;

(iii) Assume that $d(f, g)>d(f, h)+d(h, g)$ holds for some $f, g, h \in \mathcal{X}$. Then, by the definition, there exists an $x_{0} \in I$ with

$$
\begin{aligned}
& \max _{0 \leq i \leq n-1}\left|f^{(i)}\left(x_{0}\right)-g^{(i)}\left(x_{0}\right)\right| \\
> & (d(f, h)+d(h, g)) \varphi\left(x_{0}\right) \\
= & d(f, h) \varphi\left(x_{0}\right)+d(h, g) \varphi\left(x_{0}\right) \\
\geq & \max _{0 \leq i \leq n-1}\left|f^{(i)}\left(x_{0}\right)-h^{(i)}\left(x_{0}\right)\right|+\max _{0 \leq i \leq n-1}\left|g^{(i)}\left(x_{0}\right)-h^{(i)}\left(x_{0}\right)\right|,
\end{aligned}
$$

but

$$
\begin{aligned}
& \left|f^{(i)}\left(x_{0}\right)-g^{(i)}\left(x_{0}\right)\right| \\
\leq & \left|f^{(i)}\left(x_{0}\right)-h^{(i)}\left(x_{0}\right)\right|+\left|g^{(i)}\left(x_{0}\right)-h^{(i)}\left(x_{0}\right)\right| \\
\leq & \max _{0 \leq i \leq n-1}\left|f^{(i)}\left(x_{0}\right)-h^{(i)}\left(x_{0}\right)\right|+\max _{0 \leq i \leq n-1}\left|g^{(i)}\left(x_{0}\right)-h^{(i)}\left(x_{0}\right)\right|
\end{aligned}
$$


for every $i \in\{0,1, \ldots, n-1\}$, which is a contradiction.

We will now prove that $(\mathcal{X}, d)$ is complete. Let $\left\{h_{k}\right\}_{k}$ be a Cauchy sequence in $(\mathcal{X}, d)$. Then, for any $\varepsilon>0$, there exists an integer $N_{\varepsilon}>0$ such that $d\left(h_{m}, h_{l}\right) \leq \varepsilon$ for all $m, l \geq N_{\varepsilon}$. It further follows from the definition of $d$ that $\forall \varepsilon>0, \exists N_{\varepsilon} \in \mathbb{N}$ such that for all $m, l \geq N_{\varepsilon}$ and every $x \in I$, $\max _{0 \leq i \leq n-1}\left|h_{m}^{(i)}(x)-h_{l}^{(i)}(x)\right| \leq \varepsilon \varphi(x)$. If $x$ is fixed, we have $\left\{h_{k}^{(i)}(x)\right\}_{k}$ is a Cauchy sequence in $\mathbb{R}$ for each $i \in\{0,1, \ldots, n-1\}$. Since $\mathbb{R}$ is complete, for every fixed $i,\left\{h_{k}^{(i)}(x)\right\}_{k}$ converges for each fixed $x \in I$. Thus, we can define a function $H_{i}: I \rightarrow \mathbb{R}, i \in\{0,1, \ldots, n-1\}$, by

$$
H_{i}(x):=\lim _{k \rightarrow \infty} h_{k}^{(i)}(x) .
$$

Since $\varphi$ is bounded on $I,\left\{h_{k}^{(i)}\right\}_{k}$ converges uniformly to $H_{i}$ for each $i \in$ $\{0,1, \ldots, n-1\}$. Hence, $H_{i}(x), i \in\{0,1, \ldots, n-1\}$, is continuous and

$$
H_{i}^{\prime}(x)=H_{i+1}(x)
$$

for $i \in\{0,1, \ldots, n-2\}$, which implies that $H_{0}$ is $(n-1)$ times continuously differentiable, and hence, $(\mathcal{X}, d)$ is complete since $d\left(h_{k}, H_{0}\right) \rightarrow 0$ as $k \rightarrow \infty$.

We should give the definitions of the Hyers-Ulam stability and the generalized Hyers-Ulam stability of Eq. (1.1).

Definition 2.2. We say that Eq. (1.1) has the Hyers-Ulam stability if there exists a constant $K>0$ with the following property:

For every $\varepsilon>0, y(x) \in C^{n}(I, \mathbb{R})$, if

$$
\left|y^{(n)}-F\left(x, y, y^{\prime}, \ldots, y^{(n-1)}\right)\right| \leq \varepsilon,
$$

then there exists some $u(x) \in C^{n}(I, \mathbb{R})$ satisfying the corresponding equation such that $|y(x)-u(x)| \leq K \varepsilon$. We call such $K$ a Hyers-Ulam stability constant for Eq. (1.1).

Definition 2.3. We say that Eq. (1.1) has the generalized Hyers-Ulam stability if it has the following properties:

For every positive continuous function $\varphi(x)$ and $y(x) \in C^{n}(I, \mathbb{R})$, if

$$
\left|y^{(n)}-F\left(x, y, y^{\prime}, \ldots, y^{(n-1)}\right)\right| \leq \varphi(x),
$$

then there exists some $u(x) \in C^{n}(I, \mathbb{R})$ satisfying the corresponding equation such that $|y(x)-u(x)| \leq \Phi(x)$, where $\Phi(x)$ is a function not depending on $f$ and $u$ explicitly.

For a given $c \in I$, we define an operator

$$
(V f)(x):=\int_{c}^{x} f(\tau) d \tau
$$

for all $x \in I$ and $f \in C(I, \mathbb{R})$. We remark that $V$ depends on $c$. 
Lemma 2.4. Let $K$ and $L$ be positive constants with $0<K L<1$. Assume that $F: I \times \mathbb{R}^{n} \rightarrow \mathbb{R}$ is a continuous function which satisfies a Lipschitz condition

$$
\left|F\left(x, y_{11}, \ldots, y_{1 n}\right)-F\left(x, y_{21}, \ldots, y_{2 n}\right)\right| \leq L \cdot \max _{1 \leq i \leq n}\left|y_{1 i}-y_{2 i}\right|
$$

for any $x \in I$ and $y_{1 i}, y_{2 i} \in \mathbb{R}, i \in\{1,2, \ldots, n\}$. If an n-times continuously differentiable function $y(x)$ satisfies

$$
\left|y^{(n)}(x)-F\left(x, y(x), y^{\prime}(x), \ldots, y^{(n-1)}(x)\right)\right| \leq \varphi(x)
$$

for each $x \in I$, where $\varphi: I \rightarrow(0, \infty)$ is a continuous function with

$$
\left|\left(V^{i} \varphi\right)(x)\right| \leq K \varphi(x)
$$

for each $x \in I$ and $i \in\{1,2, \ldots, n\}$, then there exists an $n$ times continuously differentiable function $u: I \rightarrow \mathbb{R}$ which satisfies $E q$. (1.1) and

$$
|y(x)-u(x)| \leq \frac{\tilde{K}}{1-K L} \varphi(x)
$$

for all $x \in I$, where $\tilde{K}$ is a positive constant.

Proof. We define operators $\Lambda_{j}$ inductively by

$$
\begin{aligned}
\left(\Lambda_{1} f\right)(x) & :=\int_{c}^{x} F\left(\tau, f(\tau), f^{\prime}(\tau), \ldots, f^{(n-1)}(\tau)\right) d \tau+w_{1}, \\
\left(\Lambda_{i} f\right)(x) & :=\int_{c}^{x}\left(\Lambda_{i-1} f\right)(\tau) \tau+w_{i}
\end{aligned}
$$

for all $f \in \mathcal{X}$ and $i \in\{2,3, \ldots, n\}$, where $w_{i}, i \in\{1,2, \ldots, n\}$, is an arbitrary given real number. Then, we define $\Lambda:=\Lambda_{n}$ and it is easy to prove that $\Lambda y \in \mathcal{X}$.

For any $y_{1}, y_{2} \in \mathcal{X}$, let $C_{y_{1} y_{2}}=d\left(y_{1}, y_{2}\right)$, that is,

$$
\left|y_{1}^{(i)}(x)-y_{2}^{(i)}(x)\right| \leq C_{y_{1} y_{2}} \varphi(x)
$$

for any $x \in I$ and $i \in\{0,1, \ldots, n-1\}$. It then follows from (2.1), (2.3), (2.4), and the definitions of $d$ and $\Lambda$ that

$$
\begin{aligned}
&\left|\left(\Lambda y_{1}\right)^{(i)}(x)-\left(\Lambda y_{2}\right)^{(i)}(x)\right| \\
&=\left|V^{n-i} \circ\left(F\left(\cdot, y_{1}(\cdot), y_{1}^{\prime}(\cdot), \ldots, y_{1}^{(n-1)}(\cdot)\right)-F\left(\cdot, y_{2}(\cdot), y_{2}^{\prime}(\cdot), \ldots, y_{2}^{(n-1)}(\cdot)\right)\right)(x)\right| \\
& \leq\left|\left(V^{n-i} \circ\left|F\left(\cdot, y_{1}(\cdot), y_{1}^{\prime}(\cdot), \ldots, y_{1}^{(n-1)}(\cdot)\right)-F\left(\cdot, y_{2}(\cdot), y_{2}^{\prime}(\cdot), \ldots, y_{2}^{(n-1)}(\cdot)\right)\right|\right)(x)\right| \\
& \leq L \cdot\left|\left(V^{n-i} \circ \max _{0 \leq i \leq n-1}\left|y_{1}^{(i)}(\cdot)-y_{2}^{(i)}(\cdot)\right|\right)(x)\right| \\
& \leq L C_{y_{1} y_{2}}\left|\left(V^{n-i} \circ \varphi(\cdot)\right)(x)\right| \\
& \leq K L C_{y_{1} y_{2}} \varphi(x) \\
& \text { for all } x \in I \text { and } i \in\{0,1, \ldots, n-1\}, \text { that is, } d\left(\Lambda y_{1}, \Lambda y_{2}\right) \leq K L C_{y_{1} y_{2}} \text {. Hence, } \\
& \text { we can conclude that } d\left(\Lambda y_{1}, \Lambda y_{2}\right) \leq K L d\left(y_{1}, y_{2}\right) \text { for any } y_{1}, y_{2} \in \mathcal{X}, \text { where we } \\
& \text { note that } 0<K L<1 .
\end{aligned}
$$


It follows from the assumptions that for an arbitrary $y_{0} \in \mathcal{X}$, there exists a constant $0 \leq C<\infty$ with

$$
\max _{0 \leq i \leq n-1}\left|\left(\Lambda y_{0}\right)^{(i)}(x)-y_{0}^{(i)}(x)\right| \leq C \varphi(x)
$$

for all $x \in I$, since $F\left(\cdot, y_{0}(\cdot), y_{0}^{\prime}(\cdot), \ldots, y_{0}^{(n-1)}(\cdot)\right)$ and $y_{0}^{(i)}(x), i \in\{0,1, \ldots$, $n-1\}$, are bounded on $I$ and $\min _{x \in I} \varphi(x)>0$. Thus, we have

$$
d\left(\Lambda y_{0}, y_{0}\right)<\infty \text {. }
$$

Therefore, according to Theorem 1.3, there exists an $(n-1)$ times continuously differentiable function $u \in \mathcal{X}$ such that $\Lambda^{k} y_{0} \rightarrow u$ as $k \rightarrow \infty$ and

$$
(\Lambda u)(x)=u(x)
$$

for each $x \in I$. Consequently, $u$ is a solution to (1.1).

To prove the uniqueness, we will now verify that $\left\{g \in \mathcal{X} \mid d\left(y_{0}, g\right)<\infty\right\}=$ $\mathcal{X}$. For any $g \in \mathcal{X}$, since $g^{(i)}$ is bounded on $I$ for all $i \in\{0,1, \ldots, n-1\}$ and $\min _{x \in I} \varphi(x)>0$, there exists a constant $0<C_{g}<\infty$ such that

$$
\left|g^{(i)}(x)-y_{0}^{(i)}(x)\right| \leq C_{g} \varphi(x)
$$

for any $x \in I$ and all $i \in\{0,1, \ldots, n-1\}$. Hence, we have $d\left(g, y_{0}\right)<\infty$ for all $g \in \mathcal{X}$. Then, $\left\{g \in \mathcal{X} \mid d\left(y_{0}, g\right)<\infty\right\}=\mathcal{X}$, which implies that $u$ is the unique solution of $\Lambda u=u$ in $\mathcal{X}$.

On the other hand, it follows from (2.2) that

$$
-\varphi(x) \leq y^{(n)}(x)-F\left(x, y(x), y^{\prime}(x), \ldots, y^{(n-1)}(x)\right) \leq \varphi(x)
$$

for all $x \in I$. If we integrate each term in the above inequality from $c$ to $x$ for $i$ times, then we obtain that

$$
\begin{aligned}
& \left|\left(V^{i} \circ y^{(n)}\right)(x)-\left(V^{i} \circ F\left(\cdot, y(\cdot), y^{\prime}(\cdot), \ldots, y^{(n-1)}(\cdot)\right)\right)(x)\right| \\
= & \mid y^{(n-i)}(x)-\left(V^{i} \circ F\left(\cdot, y(\cdot), y^{\prime}(\cdot), \ldots, y^{(n-1)}(\cdot)\right)\right)(x) \\
& \quad-\sum_{k=1}^{i}\left(V^{i-k} \circ y^{(n-k)}(c)\right)(x) \mid \\
\leq & \left|\left(V^{i} \varphi\right)(x)\right|
\end{aligned}
$$

for any $x \in I$ and $i \in\{1,2, \ldots, n\}$, where $V^{0}$ denotes identity mapping. Recall the definition of $\Lambda$, and thus

$$
\begin{aligned}
\left|y^{(i)}(x)-(\Lambda y)^{(i)}(x)\right| & \leq\left|\left(V^{n-i} \varphi\right)(x)\right|+\sum_{k=1}^{n-i}\left(V^{n-i-k} \circ\left|\omega_{k}-y^{(n-k)}(c)\right|\right)(x) \\
& \leq K \varphi(x)+\sum_{k=1}^{n-i}\left(V^{n-i-k} \circ\left|\omega_{k}-y^{(n-k)}(c)\right|\right)(x)
\end{aligned}
$$

for each $x \in I$ and $i \in\{0,1, \ldots, n-1\}$. 
Since for any $i \in\{0,1, \ldots, n-1\}$,

$$
\sum_{k=1}^{n-i}\left(V^{n-i-k} \circ\left|\omega_{k}-y^{(n-k)}(c)\right|\right)(x)
$$

is bounded on $I$ and $\min _{x \in I} \varphi(x)>0$, there exists a positive constant $\tilde{K}\left(K, \omega_{1}\right.$, $\left.\omega_{2}, \ldots, \omega_{n}\right)<\infty$ such that

$$
d(y(x),(\Lambda y)(x)) \leq \tilde{K}
$$

Finally, Theorem 1.3 together with the above inequality implies that

$$
d(y, u) \leq \frac{1}{1-K L} d(y, \Lambda y) \leq \frac{\tilde{K}}{1-K L},
$$

which completes our proof.

Remark 2.5. Function $u$ is the unique solution of Eq. (1.1) with $u^{(n-i)}(c)=\omega_{i}$ for every $i \in\{0,1, \ldots, n-1\}$.

Remark 2.6. When $\omega_{i}=y^{(i)}(c)$ for each $i \in\{0,1, \ldots, n-1\}, u(x)$ is the unique solution of Eq. (1.1) satisfying $u^{(i)}(c)=y^{(i)}(c)$ for each $i \in\{0,1, \ldots, n-1\}$ with

$$
|y(x)-u(x)| \leq \frac{K}{1-K L} \varphi(x) .
$$

Given real numbers $a$ and $b$, let $I$ denote either $(-\infty, b]$ or $\mathbb{R}$ or $[a, \infty)$. Set either $c=a$ for $I=[a, \infty)$ or $c=b$ for $I=(-\infty, b]$ or $c$ is a fixed real number if $I=\mathbb{R}$. By using the same technique from [23], we can prove the theorem for the case of unbounded intervals.

Corollary 2.7. Assume that $F: I \times \mathbb{R}^{n} \rightarrow \mathbb{R}$ is a continuous function which satisfies the Lipschitz condition (2.1). If an n-times continuously differentiable function $y(x)$ satisfies inequality (2.2) for each $x \in I$, then there exists an $n$ times continuously differentiable function $u: I \rightarrow \mathbb{R}$ which satisfies Eq. (1.1) and

$$
|y(x)-u(x)| \leq \frac{K}{1-K L} \varphi(x)
$$

for all $x \in I$, where $\varphi$ satisfies condition (2.3) on $I$.

The following theorem is the main theorem of this paper which shows that condition (2.3) is not necessary.

Theorem 2.8. Assume that $F: I \times \mathbb{R}^{n} \rightarrow \mathbb{R}$ is a continuous function which satisfies the Lipschitz condition (2.1). If an n-times continuously differentiable function $y(x)$ satisfies

$$
\left|y^{(n)}(x)-F\left(x, y(x), y^{\prime}(x), \ldots, y^{(n-1)}(x)\right)\right| \leq \varphi(x)
$$


for each $x \in I$, where $\varphi: I \rightarrow(0, \infty)$ is a continuous function, then there exists an $n$ times continuously differentiable function $u: I \rightarrow \mathbb{R}$ which satisfies Eq. (1.1) and

$$
|y(x)-u(x)| \leq \frac{\tilde{K}(x)}{1-K L} \varphi(x)
$$

for all $x \in I$, where $\tilde{K}(x)$ is a nonnegative function depending on $\varphi(x)$ only.

Proof. Let $K$ be an arbitrary positive number with $K L<1$. Since $\varphi(x)>0$ for every $x \in I$, we have $\sup _{x \in I} \varphi(x)<\infty$ and $\inf _{x \in I} \varphi(x)>0$. Then for any given constant $c \in[a, b]$, there exists a $\delta>0$ not depending on $c$ such that

$$
\left|\left(V^{i} \circ \varphi\right)(x)\right| \leq \delta^{i} \sup _{x \in I} \varphi(x) \leq K \inf _{x \in I} \varphi(x) \leq K \varphi(x)
$$

for every $x \in[c-\delta, c+\delta] \cap I$ and $i \in\{1,2, \ldots, n\}$. (Operator $V$ depends on $c$.)

Let $\left\{a_{0}, a_{1}, \ldots, a_{m}\right\}$ be a partition of interval $I$ with the properties:

(i) $a_{0}=a, a_{m}=b$, and $0<a_{i}-a_{i-1} \leq \delta$ for $i \in\{1,2, \ldots, m\}$;

(ii) $I_{i}=\left[a_{i-1}, a_{i}\right]$ for $i \in\{1,2, \ldots, m\}$.

According to Lemma 2.4 and Remark 2.6, there exists a unique $n$ times continuously differentiable function $u_{1}(x)$ satisfying Eq. (1.1) and $u_{1}^{(k)}\left(a_{0}\right)=$ $y^{(k)}\left(a_{0}\right)$ for each $k \in\{0,1, \ldots, n-1\}$ with

for every $x \in I_{1}$.

$$
\left|y(x)-u_{1}(x)\right| \leq \frac{K}{1-K L} \varphi(x)
$$

Similarly, by Lemma 2.4 and Remark 2.5, we obtain a unique solution $u_{i} \in \mathcal{X}$ on $I_{i}, i \in\{1,2, \ldots, m\}$, such that

$$
|y(x)-u(x)| \leq \frac{\tilde{K}_{i}}{1-K L} \varphi(x)
$$

for every $x \in I_{i}$ and

$$
u_{i}^{(k)}\left(a_{i-1}\right)=u_{i-1}^{(k)}\left(a_{i-1}\right)
$$

for every $i \in\{2,3, \ldots, m\}$ and $k \in\{0,1, \ldots, n-1\}$. Moreover, since

$$
u_{i}^{(n)}(x)=F\left(x, u_{i}^{\prime}(x), u_{i}^{\prime \prime}(x), \ldots, u_{i}^{(n-1)}(x)\right),
$$

we obtain

$$
u_{i}^{(n)}\left(a_{i-1}\right)=u_{i-1}^{(n)}\left(a_{i-1}\right)
$$

for all $i \in\{2,3, \ldots, m\}$.

Now, we define $u: I \rightarrow \mathbb{R}$ by $u(x)=u_{i}(x)$ on $I_{i}$ for each $i \in\{1,2, \ldots, m\}$. The function is well defined and is $n$ times continuously differentiable.

If we define $\tilde{K}(x):=\tilde{K}_{i}$ on $I_{i}$ for each $i \in\{1,2, \ldots, m\}$, we have

$$
|y(x)-u(x)| \leq \frac{\tilde{K}(x)}{1-K L} \varphi(x)
$$


for each $x \in I$, which completes the proof.

Let $I$ denote either $(-\infty, b]$ or $\mathbb{R}$ or $[a, \infty)$. We obtain the theorem for the case of unbounded intervals.

Corollary 2.9. Assume that $F: I \times \mathbb{R}^{n} \rightarrow \mathbb{R}$ is a continuous function which satisfies the Lipschitz condition (2.1), $\varphi: I \rightarrow(0, \infty)$ is a continuous function. Then Eq. (1.1) has the generalized Hyers-Ulam stability.

Proof. It is not difficult to prove this corollary by using a similar technique of the proof of Theorem 3.2 in [23] and the result given in Theorem 2.8 in this paper.

As a corollary of Theorem 2.8, we prove the Hyers-Ulam stability of the differential equation (1.1) on a finite closed interval.

Corollary 2.10. Let $I=[a, b]$ and let $L$ be positive constant. Assume that $F: I \times \mathbb{R}^{n} \rightarrow \mathbb{R}$ is a continuous function which satisfies the Lipschitz condition (2.1) for any $x \in I$. If an $n$ times continuously differentiable function $y \in \mathcal{X}$ satisfies

$$
\left|y^{(n)}(x)-F\left(x, y(x), y^{\prime}(x), \ldots, y^{(n-1)}(x)\right)\right| \leq \varepsilon
$$

for each $x \in I$ and for some $\varepsilon \geq 0$, then Eq. (1.1) has the Hyers-Ulam stability on $I$.

Proof. Let $\varphi(x)=\varepsilon, K=\frac{1}{2 L}$, and let $\delta=\delta(K)=\min \{\sqrt[n]{K}, K\}=\min \left\{\frac{1}{\sqrt[n]{2 L}}\right.$, $\left.\frac{1}{2 L}\right\}$. Hence, according to the proof of Lemma 2.4 and Theorem 2.8, we can conclude that there exists an $n$ times continuously differentiable function $u$ : $I \rightarrow \mathbb{R}$ which satisfies Eq. (1.1) and

$$
|y(x)-u(x)| \leq \frac{\left(2 \frac{\delta^{n}-1}{\delta-1}\right)^{[(b-a) / \delta]+1}-1}{\frac{\delta^{n}-1}{\delta-1} 2 L-L} \varepsilon
$$

for every $x \in I$, where $[(b-a) / \delta]=\max \{n \in \mathbb{Z} \mid n<(b-a) / \delta\}$. This completes our proof. (When $\varepsilon=0, y$ is a solution of Eq. (1.1).)

Remark 2.11. Indeed, $2 L$ can be replaced with $p L$ in Corollary 2.10 , where $p$ is an arbitrary number larger than 1 . We can conclude that the Hyers-Ulam stability constant of Eq. (1.1) is not larger than

$$
\inf _{p>1} \frac{\left(\frac{p}{p-1} \frac{\delta^{n}-1}{\delta-1}\right)^{[(b-a) / \delta]+1}-1}{\frac{\delta^{n}-1}{\delta-1} p L-(p L-L)},
$$

where $\delta=\min \left\{\frac{1}{\sqrt[n]{p L}}, \frac{1}{p L}\right\}$ and $[(b-a) / \delta]=\max \{n \in \mathbb{Z} \mid n<(b-a) / \delta\}$. (We remark that the solution $u(x)$ of Eq. (1.1) does not change as $p$ changes.) 
Remark 2.12. When $n=1$, we answer the question raised by Jung [23, Section 4]). That is, if $I=[a, b]$ with $-\infty<a<b<\infty$ and $F$ satisfies the Lipschitz condition, then the differential equation $y^{\prime}=F(x, y)$ has the Hyers-Ulam stability with a Hyers-Ulam stability constant no larger than

$$
\liminf _{p \rightarrow \infty} \frac{\left(\frac{p}{p-1}\right)^{[p L(b-a)]+1}-1}{L} \rightarrow \frac{e^{L(b-a)}-1}{L}
$$

and has the generalized Hyers-Ulam stability provided $I$ is either $(-\infty, b]$ or $\mathbb{R}$ or $[a, \infty)$. (It is not difficult to show that there are some differential equations of first order satisfying a Lipschitz condition which do not have the Hyers-Ulam stability on unbounded interval, e.g. $y^{\prime}=0$.)

Remark 2.13. $\frac{e^{L(b-a)}-1}{L}$ is not necessarily the best Hyers-Ulam stability constant of a differential equation of first order with the Lipschitz condition, e.g. $y^{\prime}=y[1]$. For more detail concerning the best Hyers-Ulam stability constant, we refer to [13].

Remark 2.14. When Lipschitz constant $L=0$, Eq. (1.1) is equivalent to

$$
y^{(n)}(x)=f(x),
$$

where $f:[a, b] \rightarrow \mathbb{R}$ is a continuous function. Then for an $n$ times continuously differentiable function $y:[a, b] \rightarrow \mathbb{R}$ with

$$
\left|y^{(n)}(x)-f(x)\right| \leq \varepsilon
$$

for each $x \in[a, b]$ and for some $\varepsilon \geq 0$, there exists an $n$ times continuously differentiable function $y_{0}:[a, b] \rightarrow \mathbb{R}$ satisfying the corresponding equation with

$$
\left|y(x)-y_{0}(x)\right| \leq\left(\frac{b-a}{2}\right)^{n} \varepsilon .
$$

Proof. We can define an $n$ times continuously differentiable function $y_{0}:[a, b]$ $\rightarrow \mathbb{R}$, which is a solution of Eq. (2.6) with the initial condition $y_{0}^{(i)}\left(\frac{a+b}{2}\right)=$ $y^{(i)}\left(\frac{a+b}{2}\right)$ for each $i \in\{0,1, \ldots, n-1\}$, then

$$
\begin{aligned}
\left|y(x)-y_{0}(x)\right| & \leq\left|\int_{\frac{a+b}{2}}^{x} \cdots \int_{\frac{a+b}{2}}^{\tau_{2}} y^{(n)}\left(\tau_{1}\right)-y_{n}^{(n)}\left(\tau_{1}\right) d \tau_{1} \cdots d \tau_{n}\right| \\
& \leq \int_{\frac{a+b}{2}}^{x} \cdots \int_{\frac{a+b}{2}}^{\tau_{2}}\left|y^{(n)}\left(\tau_{1}\right)-y_{0}^{(n)}\left(\tau_{1}\right)\right| d \tau_{1} \cdots d \tau_{n} \\
& \leq \int_{\frac{a+b}{2}}^{x} \cdots \int_{\frac{a+b}{2}}^{\tau_{2}}\left|y^{(n)}\left(\tau_{1}\right)-f_{0}^{(n)}\left(\tau_{1}\right)\right| d \tau_{1} \cdots d \tau_{n} \\
& \leq\left(\frac{b-a}{2}\right)^{n} \varepsilon
\end{aligned}
$$

for every $x \in[a, b]$, which completes our proof. 
By using Theorem 2.8, we can obtain the following corollary which was proved by Rezaei and Jung in [44].

Corollary 2.15. If for any $i \in\{0,1, \ldots, n\}, a_{i}(x)$ is a continuous function on $I$, then the linear differential equation

$$
y^{(n)}(x)+a_{n}(x) y^{n-1}(x)+\cdots+a_{2}(x) y^{\prime}(x)+a_{1}(x) y(x)+a_{0}(x)=0
$$

has the Hyers-Ulam stability on I. (The above equation has the generalized Hyers-Ulam stability when I is an unbounded interval.)

We investigated the stability of nonlinear equations of the form (1.1) in this paper, it is an open problem that whether it is possible to find some condition to ensure that the Hyers-Ulam stability holds for the case of general nonlinear differential equations in the form of

$$
F\left(y^{(n)}, y^{(n-1)}, \ldots, y^{\prime}, y, x\right)=0 .
$$

Acknowledgements. This work was supported by the National Natural Science Foundation of China (10871213).

\section{References}

[1] C. Alsina and R. Ger, On some inequalities and stability results related to the exponential function, J. Inequal. Appl. 2 (1998), no. 4, 373-380.

[2] T. Aoki, On the stability of the linear transformation in Banach spaces, J. Math. Soc. Japan 2 (1950), 64-66.

[3] J. Baker, J. Lawrence, and F. Zorzitto, The stability of the equation $f(x+y)=f(x) f(y)$, Proc. Amer. Math. Soc. 74 (1979), no. 2, 242-246.

[4] D. G. Bourgin, Classes of transformations and bordering transformations, Bull. Amer. Math. Soc. 57 (1951), 223-237.

[5] M. Burger, N. Ozawa, and A. Thom, On Ulam stabiltity, Israel J. Math (2012), 1-21.

[6] K. Ciepliński, Applications of fixed point theorems to the Hyers-Ulam stability of functional equations - a survey, Ann. Func. Anal. 3 (2012), no. 1, 151-164.

[7] J. B. Diaz and B. Margolis, A fixed point theorem of the alternative for contractions on a generalized complete metric space, Bull. Amer. Math. Soc. 74 (1968), 305-309.

[8] V. A. Faiziev, Th. M. Rassias, and P. K. Sahoo, The space of $(\psi, \gamma)$-additive mappings on semigroups, Trans. Amer. Math. Soc. 354 (2002), no. 11, 4455-4472.

[9] G. L. Forti, Hyers-Ulam stability of functional equations in several variables, Aequationes Math. 50 (1995), no. 1-2, 143-190.

[10] Z. Gajda, On stability of additive mappings, Internat. J. Math. Math. Sci. 14 (1991), 431-434.

[11] P. Găvruţă, A generalization of the Hyers-Ulam-Rassias stability of approximately additive mappings, J. Math. Anal. Appl. 184 (1994), no. 3, 431-436.

[12] P. Găvruţă, S.-M. Jung, and Y. Li, Hyers-Ulam stability for second-order linear differential equations with boundary conditions, Electron. J. Diff. Equ. 2011 (2011), no. 80, $1-5$.

[13] O. Hatori, K. Kobayasi, T. Miura, H. Takagi, and S. E. Takahasi, On the best constant of Hyers-Ulam stability, J. Nonlinear Convex Anal. 5 (2004), no. 3, 387-393.

[14] T. Huuskonen and J. Väisälä, Hyers-Ulam constants of Hilbert spaces, Studia Math. 153 (2002), no. 1, 31-40.

[15] D. H. Hyers, On the stability of the linear functional equation, Proc. Nat. Acad. Sci. U.S.A. 27 (1941), 222-224. 
[16] D. H. Hyers and S. M. Ulam, On approximate isometries, Bull. Amer. Math. Soc. 51 (1945), 288-292.

[17] _ Approximate isometries of the space of continuous functions, Ann. Math. 48 (1947), 285-289.

[18] On the stability of differential expressions, Math. Mag. 28 (1954), 59-64.

[19] K.-W. Jun and Y.-H. Lee, A generalization of the Hyers-Ulam-Rassias stability of Jensen's equation, J. Math. Anal. Appl. 238 (1999), no. 1, 305-315.

[20] S.-M. Jung, Hyers-Ulam stability of linear differential equations of first order, Appl. Math. Lett. 17 (2004), no. 10, 1135-1140.

[21] _ Hyers-Ulam stability of linear differential equations of first order. III, J. Math. Anal. Appl. 311 (2005), no. 1, 139-146.

[22] _ Hyers-Ulam stability of linear differential equations of first order. II, Appl. Math. Lett. 19 (2006), no. 9, 854-858.

[23] — A fixed point approach to the stability of differential equations $y^{\prime}=F(x, y)$, Bull. Malays. Math. Sci. Soc. (2) 33 (2010), no. 1, 47-56.

[24] S.-M. Jung, D. Popa, and M. Th. Rassias, On the stability of the linear functional equation in a single variable on complete metric groups, J. Global Optimi. 59 (2014), no. 1, 165-171.

[25] Pl. Kannappan, Functional Equations and Inequalities with Applications, Springer, New York, 2009.

[26] Y.-H. Lee, S.-M. Jung, and M. Th. Rassias, On an n-dimensional mixed type additive and quadratic functional equation, Appl. Math. Comput. 228 (2014), 13-16.

[27] Y. Li, Hyers-Ulam stability of linear differential equations $y^{\prime \prime}=\lambda^{2} y$, Thai J. Math. 8 (2010), no. 2, 215-219.

[28] Y. Li and Y. Shen, Hyers-Ulam stability of nonhomogeneous linear differential equations of second order, Internat. J. Math. Math. Sci 2009 (2009), Article ID 576852, 7 pp.

[29] - Hyers-Ulam stability of linear differential equations of second order, Appl. Math. Lett. 23 (2010), no. 3, 306-309.

[30] T. Miura, On the Hyers-Ulam stability of a differentiable map, Sci. Math. Jpn. 55 (2002), no. 1, 17-24.

[31] T. Miura, S.-M. Jung, and S.-E. Takahasi, Hyers-Ulam-Rassias stability of the Banach space valued linear differential equations $y^{\prime}=\lambda y$, J. Korean Math. Soc. 41 (2004), no. 6, 995-1005.

[32] T. Miura, M. Miyajima, and S.-E. Takahasi, Hyers-Ulam stability of linear differential operator with constant coefficients, Math. Nachr. 258 (2003), 90-96.

[33] T. Miura, H. Oka, S.-E. Takahasi, and N. Niwa, Hyers-Ulam stability of the first order linear differential equation for Banach space-valued holomorphic mappings, J. Math. Inequal. 3 (2007), no. 3, 377-385.

[34] M. Obłoza, Hyers stability of the linear differential equation, Rocznik Nauk.-Dydakt. Prace Mat. 13 (1993), 259-270.

[35] Connections between Hyers and Lyapunov stability of the ordinary differential equations, Rocznik Nauk.-Dydakt. Prace Mat. 14 (1997), 141-146.

[36] M. Omladič and P. Šemrl, On non linear perturbations of isometries, Math. Ann. 303 (1995), no. 1, 617-628.

[37] C.-G. Park, On the stability of the linear mapping in Banach modules, J. Math. Anal. Appl. 275 (2002), no. 2, 711-720.

[38] D. Popa and I. Rașa, Hyers-Ulam stability of the linear differential operator with nonconstant coefficients, Appl. Math. Comput. 219 (2012), no. 4, 1562-1568.

[39] V. Radu, The fixed point alternative and the stability of functional equations, Fixed Point Theory 4 (2003), no. 1, 91-96.

[40] Th. M. Rassias, On the stability of the linear mapping in Banach spaces, Proc. Amer. Math. Soc. 72 (1978), no. 2, 297-300. 
[41] J. M. Rassias, On approximation of approximately linear mappings by linear mappings, J. Funct. Anal. 46 (1982), no. 1, 126-130.

[42] Th. M. Rassias and P. Šemrl, On the behavior of mappings which do not satisfy HyersUlam stability, Proc. Amer. Math. Soc. 114 (1992), no. 4, 989-993.

[43] _ On the Hyers-Ulam stability of linear mappings, J. Math. Anal. Appl. 173 (1993), no. 2, 325-338.

[44] H. Rezaei and S.-M. Jung, A fixed point approach to the stability of linear differential equations, Bull. Malays. Math. Sci. Soc. (2), in press.

[45] H. Rezaei, S.-M. Jung, and Th. M. Rassias, Laplace transform and Hyers-Ulam stability of linear differential equations, J. Math. Anal. Appl. 403 (2013), no. 1, 244-251.

[46] P. K. Sahoo and Pl. Kannappan, Intoduction to Functional Equations, Chapman and Hall CRC, Boca Raton, Florida, 2011.

[47] P. Šemrl and J. Väisälä, Nonsurjective nearisometries of Banach spaces, J. Funct. Anal. 198 (2003), no. 1, 268-278.

[48] S.-E. Takahasi, T. Miura, and S. Miyajima, On the Hyers-Ulam stability of the Banach space-valued differential equation $y^{\prime}=\lambda y$, Bull. Korean Math. Soc. 39 (2002), no. 2, 309-315.

[49] S. M. Ulam, A Collection of the Mathematical Problems, Interscience, New York, 1960.

JiNGHAO HUANG

Department of Mathematics

Sun YAT-SEN UNIVERSITY

Guangzhou, 510275, P. R. China

E-mail address: hjinghao@mail2.sysu.edu.cn

SOON-MO Jung

Mathematics Section

College of Science And Technology

HONGIK UNIVERSITY

SEJONG 339-701, KoreA

E-mail address: smjung@hongik.ac.kr

YONGJIN LI

Department of Mathematics

Sun YAT-SEN UNIVERSITY

Guangzhou, 510275, P. R. China

E-mail address: stslyj@mail.sysu.edu.cn 